\title{
UN ENFOQUE DEL APRENDIZAJE BASADO EN PROBLEMAS (ABP) COMO METODOLOGÍA DIDÁCTICA EN LAS PRÁCTICAS EXTRACURRICULARES DEL GRADO EN CONSERVACIÓN-RESTAURACIÓN
}

\section{A Problem-Based Learning (PBL) approach as a teaching methodology in extracurricular work experience for the Degree in Conservation and Restoration}

\author{
Antonio J. SÁnCHEZ FernándeZ \\ Universidad de La Laguna \\ Correo-e: asanchez@ull.edu.es
}

Recibido: I8 de agosto de 2020

Envío a informantes: I2 de septiembre de 2020

Aceptación definitiva: 27 de febrero de 202I

RESUMEN: El presente artículo expone la experiencia práctica extracurricular en el Grado de Conservación y Restauración de Bienes Culturales de la Universidad de Sevilla, con la restauración del retablo de la ermita de la Virgen de Valme (Dos Hermanas, Sevilla). El objetivo fundamental de este programa fue la profundización de los conocimientos adquiridos en el aula y la aplicación de metodologías específicas de intervención en bienes culturales en un entorno laboral real y con un enfoque del Aprendizaje Basado en Problemas (ABP). El desarrollo práctico de la intervención de restauración supuso el entrenamiento y mejora de las habilidades profesionales de los alumnos, asociadas a la superación de fases conectadas de forma dependiente, un aprendizaje autodirigido y unos resultados autosupervisados. Así, manifestamos la necesidad de recursos didácticos apropiados para las especificidades de la disciplina de la conservación-restauración. tario.

Palabras Clave: alumno en prácticas; aprendizaje por experiencia; grado universi- 
Aвstract: This article describes the extracurricular work experience for the Degree in Conservation and Restoration of Cultural Assets at the University of Seville, focusing on the restoration of the altarpiece of the Chapel of the Virgin of Valme (Dos Hermanas, Seville). The key goal of this programme was to further explore the knowledge acquired in the lecture hall and to apply specific methodologies for intervention in cultural assets in a real-life work environment and with a focus on Problem-Based Learning (PBL). The practical development of the restoration work involved practising and enhancing the professional skills of the students, associated with the understanding of connected phases in a dependent way, self-directed learning and self-assessed results. As a result, we underline the need for appropriate teaching resources for the specific features of the conservation-restoration discipline.

KEY WORDS: academic degree; experiential learning; trainee.

\section{Introducción}

L A CONSERVACIÓN-RESTAURACIÓN de bienes culturales es un área de conocimiento académico relativamente reciente. Requiere de una serie de competencias teóricas y prácticas, un espíritu crítico y unas destrezas técnicas. En palabras de Cesare Brandi, teórico de la disciplina, la restauración es «el momento metodológico del reconocimiento de la obra de arte, en su consistencia física y en su doble polaridad estética e histórica, en orden a su transmisión al futuro» (Brandi, 2007, p. 15). Pero ¿cómo llegamos a la competencia del reconocimiento de ese «momento metodológico» en un contexto profesional?

El interés por la innovación educativa y el replanteamiento de modelos propone el reto de la enseñanza de esas competencias al conservador-restaurador como profesional. La dificultad evidente de disponer en un aula de determinadas tipologías de bienes (Prado-Campos y Zambrana-Vega, 20I7, p. 55), como retablos o pinturas murales, hace que el programa de prácticas académicas se establezca como un sistema eficaz para complementar los planes de estudios reglados.

Así, el presente artículo expone la articulación didáctica de las prácticas extracurriculares para la restauración del retablo de la ermita de la Virgen de Valme (Dos Hermanas, Sevilla). Dichas prácticas se realizaron mediante un convenio con la Universidad de Sevilla y la Hermandad de Valme para alumnos del Grado en Conservación y Restauración de Bienes Culturales. Se lanzó una oferta para cuatro alumnos y una temporalización de 200 horas, ejecutadas entre los meses de julio y agosto de 2019 . En definitiva, las prácticas extracurriculares se entienden como un proceso formativo, que involucra a la universidad y otras instituciones externas, que ofrece una tutorización de una experiencia profesional real. Así, los alumnos adquieren conocimientos y competencias básicas propias de su futura profesión.

En este sentido, consideramos que el planteamiento metodológico del Aprendizaje Basado en Problemas (ABP) se establece como una opción adaptativa a las necesidades formativas del conservador-restaurador.

Destacamos el desarrollo de investigaciones que ponen en relación la formación en prácticas con la adquisición de competencias profesionales (Molina et al., 2004; Rodríguez, Calmaestra y Maestre, 20I5). No obstante, sería necesario profundizar en 
UN ENFOQUE DEL APRENDIZAJE BASADO EN PROBLEMAS (ABP) COMO METODOLOGÍA DIDÁCTICA EN LAS PRÁCTICAS EXTRACURRICULARES DEL GRADO... ANTONIO J. SÁNCHEZ FERNÁNDEZ

la comparación del impacto de las prácticas universitarias sobre la autopercepción de adquisición de dichas competencias (Rodríguez, Calmaestra y Maestre, 20I5, p. 4I).

\section{Marco normativo de las prácticas extracurriculares de la Universidad de Sevilla}

Las prácticas formativas en el entorno universitario están reguladas por el Real Decreto 592/20I4, que ordena los Programas de Cooperación Educativa, para incorporarlos al plan docente académico o como complemento del mismo, como es el caso de las prácticas extracurriculares.

El Secretariado de Prácticas en Empresas y Empleo de la Universidad de Sevilla depende del Vicerrectorado de Transferencia Tecnológica y se creó en abril de 20I2, como enlace entre los estudiantes y el mundo laboral. Es el responsable de la tramitación de las prácticas en empresas e instituciones. De esta forma, la Universidad de Sevilla regula las Prácticas Académicas Externas con el Acuerdo Io.I/CG 23-5-I7 (BOUs n. ${ }^{\circ} 3 / 2017$, de 14 de junio), que define las prácticas extracurriculares como «aquellas que los estudiantes podrán realizar con carácter voluntario durante su periodo de formación y que, aun teniendo los mismos fines que las prácticas curriculares, no forman parte del correspondiente Plan de Estudios» (artículo 5.b). 9.b.):

Del mismo modo, establece los requisitos de participación en el programa (artículo

- Estar matriculado en la Universidad de Sevilla en la titulación para la que se oferta la práctica.

- Haber superado el 50\% de los créditos necesarios para obtener el título (debe estar matriculado en el mimo curso académico para el que se realicen las prácticas. Si se alcanzasen el ıoo\% de los créditos, se podrían realizar si se está matriculado de al menos una asignatura reglada).

- No haber realizado prácticas con una duración igual al máximo establecido.

- No mantener ninguna relación contractual con la entidad en la que se van a realizar las prácticas.

Igualmente la entidad colaboradora debe cumplir los siguientes requisitos para participar en el programa (artículo io):

- Estar debidamente constituida.

- Disponer de los medios e instalaciones adecuadas para el desarrollo de las prácticas

- Disponer de personal cualificado para la supervisión de las prácticas.

\section{Contextualización del entorno de aprendizaje: la restauración del retablo de la ermita de Cuarto (Dos Hermanas, Sevilla)}

El retablo se ubica en el muro suroeste de la ermita de Cuarto. La ermita tiene una nave principal, de planta rectangular y varias estancias adosadas. La tradición atribuye su construcción al agradecimiento del rey Fernando II el Santo por la conquista de Sevilla. La primitiva construcción fue ampliándose a medida que crecía el peregrinaje. A mediados del s. Xviı una explosión cercana que afectó a la ermita, que fue recons- 


\section{UN ENFOQUE DEL APRENDIZAJE BASADO EN PROBLEMAS (ABP) COMO METODOLOGÍA 3 I 6 DIDÁCTICA EN LAS PRÁCTICAS EXTRACURRICULARES DEL GRADO... ANTONIO J. SÁNCHEZ FERNÁNDEZ}

truida. En el siglo XVIII, se ejecutaron obras de mejora y ampliación por el mecenazgo de la familia Rivas. En el siglo xix, a partir de la retirada y traslado de la Virgen a Dos Hermanas, comienza un periodo de declive por abandono. A mediados de dicho siglo, gracias al rescate literario de la ermita por la escritora Cecilia Böhl de Faber (Fernán Caballero), los duques de Montpensier reedificaron la capilla. El proyecto fue realizado por el arquitecto Balbino Marrón e intervinieron artistas de la confianza de los duques, como los pintores Cabral Bejarano y Juan de Lizasoain, además del arquitecto José María Ríos (Santos Gil, 2009).

El retablo presentaba unas peculiaridades históricas, estéticas y materiales que determinaban su estado de autenticidad y de conservación. Con la rehabilitación de la capilla (siglo XIX), se compró y se adaptó el retablo. Poco conocemos de su origen y ubicación primitiva, solo que procedía de la localidad de La Rinconada (Sevilla) y una cartela superior que fecha el dorado en $\mathbf{1 7 8 8}$. No obstante, en el transcurso de la intervención se documentaron elementos indicativos para determinar el aprovechamiento de partes del retablo de diferentes periodos históricos, así como los suplementos necesarios para adaptarlo a su actual ubicación. Igualmente, existió una intervención anterior realizada en la década de 1990.

Los objetivos generales de la actuación técnica fueron tanto profundizar en el conocimiento del retablo, desde una doble instancia: histórica y material, como recuperar la integridad, simbología y eficacia iconográfica de la obra, teniendo en cuenta el conjunto de valores culturales que posee el bien. La intervención técnica se basó en dos aspectos fundamentales: por un lado, un carácter conservativo, con el objetivo de paliar los daños estructurales. Y, por otro, una fase de restauración que facilitó la unidad estética del retablo y la lectura de la obra.

\section{Objetivos de las prácticas extracurriculares en la ermita de la Virgen de Valme}

El objetivo general de la práctica extracurricular fue el de complementar la capacitación del alumno como conservador-restaurador profesional. Entendemos que esta cualificación de la profesión pasa por la suficiencia en la ejecución reflexiva de la investigación, diagnóstico e intervención que necesite cada bien cultural.

Para ello, fueron necesarios unos objetivos de conocimiento teórico y otros de aplicación práctica:

- Conocer los textos normativos en materia de conservación-restauración.

- Diferenciar las técnicas y materiales constitutivos de los retablos lígneos.

- Comprender los factores de alteración/degradación e indicativos visuales.

- Distinguir procedimientos y técnicas de examen.

- Aplicar procedimientos y técnicas de tratamiento.

- Conocer y aplicar las normas de seguridad y salud.

- Aplicar herramientas para la documentación del retablo.

- Reflexionar de forma crítica el manejo de criterios de intervención (Instituto Andaluz del Patrimonio Histórico, sin fecha).

- Difundir las actuaciones (estudio e intervención) en el retablo.

En la Tabla I podemos observar qué objetivos planteamos en cada fase en la que hemos estructurado la práctica extracurricular de la restauración del retablo. 
UN ENFOQUE DEL APRENDIZAJE BASADO EN PROBLEMAS (ABP) COMO METODOLOGÍA DIDÁCTICA EN LAS PRÁCTICAS EXTRACURRICULARES DEL GRADO... ANTONIO J. SÁNCHEZ FERNÁNDEZ

TABla i. Resumen conceptual de las prácticas de restauración.

\begin{tabular}{|c|c|c|}
\hline $\begin{array}{l}\text { Definición del } \\
\text { problema }\end{array}$ & Objetivos de aprendizaje & $\begin{array}{c}\text { Fases de } \\
\text { intervención }\end{array}$ \\
\hline & $\begin{array}{l}\text { Conocimiento de técnicas y materiales } \\
\text { constitutivos de retablos lígneos. }\end{array}$ & \\
\hline $\begin{array}{l}\text { tal y como ha llegado a } \\
\text { nuestros días o } \\
\text { repristinación? }\end{array}$ & $\begin{array}{l}\text { Conocimiento de factores de } \\
\text { alteración/degradación e indicativos visuales } \\
\text { Procedimientos y técnicas de examen }\end{array}$ & $\begin{array}{l}\mathbf{1}^{\text {a }} \text { Fase. } \\
\text { Estudios } \\
\text { previos }\end{array}$ \\
\hline $\begin{array}{l}\text { ¿Qué valores culturales } \\
\text { subordino: valor } \\
\text { histórico o valor } \\
\text { estético? }\end{array}$ & $\begin{array}{l}\text { Procedimientos y técnicas de tratamiento } \\
\text { Aplicación crítica de criterios de intervención } \\
\text { Aplicación de herramientas para la } \\
\text { documentación del retablo }\end{array}$ & $\begin{array}{l}\text { (duración: } 1 \\
\text { semana) }\end{array}$ \\
\hline $\begin{array}{l}\text { Problema } 2 \\
\text { ¿Qué procedimientos y } \\
\text { técnicas de intervención } \\
\text { debemos aplicar para } \\
\text { mantener la } \\
\text { autenticidad histórica y } \\
\text { material del retablo? }\end{array}$ & $\begin{array}{l}\text { Conocimiento de los textos normativos en } \\
\text { materia de conservación-restauración } \\
\text { Conocimiento de factores de } \\
\text { alteración/degradación e indicativos visuales } \\
\text { Procedimientos y técnicas de tratamiento } \\
\text { Aplicación crítica de criterios de intervención } \\
\text { Aplicación de normas de seguridad y salud }\end{array}$ & $\begin{array}{l}\mathbf{2}^{\mathbf{a}} \text { Fase. } \\
\text { Conservación } \\
\text { (duración: } 2 \\
\text { semanas) }\end{array}$ \\
\hline $\begin{array}{l}\text { Problema } 3 \\
\text { ¿Qué procedimientos y } \\
\text { técnicas de intervención } \\
\text { debemos aplicar para } \\
\text { mantener la } \\
\text { autenticidad histórica y } \\
\text { material del retablo? }\end{array}$ & $\begin{array}{l}\text { Conocimiento de los textos normativos en } \\
\text { materia de conservación-restauración } \\
\text { Conocimiento de factores de } \\
\text { alteración/degradación e indicativos visuales } \\
\text { Procedimientos y técnicas de tratamiento } \\
\text { Aplicación crítica de criterios de intervención } \\
\text { Aplicación de normas de seguridad y salud }\end{array}$ & $\begin{array}{l}3^{\mathbf{a}} \text { Fase. } \\
\text { Restauración } \\
\text { (duración: } 3 \\
\text { semanas) }\end{array}$ \\
\hline $\begin{array}{l}\text { Problema } 4 \\
\text { Patrimonio cultural y } \\
\text { medios de } \\
\text { comunicación: ¿morir } \\
\text { de amor o morir de } \\
\text { olvido? }\end{array}$ & $\begin{array}{l}\text { Diseñar estrategias de difusión y divulgación } \\
\text { de las actividades desarrolladas }\end{array}$ & $\begin{array}{l}\mathbf{4}^{\mathbf{a}} \text { Fase. } \\
\text { Difusión }\end{array}$ \\
\hline
\end{tabular}

Nuestra propuesta de ABP del retablo de la Virgen de Valme se orienta, de forma general, hacia los objetivos enunciados por Barrows (I986) y adaptados a la idiosincrasia de la conservación-restauración de bienes culturales:

- Estructurar el conocimiento para su uso en el contexto de la conservación-restauración (Morales Bueno, 2018, p. I00; Restrepo Gómez, 2005, pp. IO-II).

- Desarrollar el aprendizaje autodirigido. Se centró en lo que hace el alumno en un contexto nuevo y en los cambios de tareas. 


\section{UN ENFOQUE DEL APRENDIZAJE BASADO EN PROBLEMAS (ABP) COMO METODOLOGÍA 3 I 8 DIDÁCTICA EN LAS PRÁCTICAS EXTRACURRICULARES DEL GRADO... ANTONIO J. SÁNCHEZ FERNÁNDEZ}

- Motivar para el aprendizaje. Los alumnos se encuentran en un proyecto que requiere de su participación directa, aumentando la motivación a medida que se observan los evidentes efectos positivos de los trabajos realizados en el retablo.

- Desarrollar las habilidades de trabajo en grupo, que conecta con la práctica profesional real que requiere de equipos de trabajo interdisciplinares.

\section{Metodología didáctica en la intervención del retablo de la ermita de Cuarto (Dos Hermanas, Sevilla)}

Los distintos enfoques de ABP coinciden en que «el punto de partida del aprendizaje debe ser un problema, cuestión o misterio que desee resolver el aprendiz» (Boud, I985, p. I3). El ABP es un método de enseñanza mediante la resolución de un problema auténtico, significativo pero desestructurado (Marra et al., 20I4, p. 22I). Para Pérez Granado (20I8, p. I64) «es una metodología que estimula el interés y la motivación del alumnado hacia el aprendizaje, los dota de más autonomía y ayuda a desarrollar el pensamiento crítico al involucrar al estudiante en un reto real». Asimismo, al gestionar el aprendizaje en un contexto real, se procura una mejora en la capacitación profesional.

El diseño de una metodología basada en el ABP debe articularse alrededor de las siguientes nociones (Gewurtz et al., 2016; Marra et al., 2014):

I. «El problema» es el centro del proceso de aprendizaje.

2. El nuevo conocimiento se apoya sobre el conocimiento previo.

3. El estudiantado autodirige su aprendizaje.

4. Trabajo en equipo con aprendizaje cooperativo.

5. Importancia de la adquisición del conocimiento frente al resultado de los procesos como aspecto secundario.

6. Análisis y reflexión sobre el aprendizaje.

7. El tutor como guía/facilitador.

Sin embargo, el problema general de la restauración del retablo es un enunciado demasiado abierto, por lo que el primer paso fue la subdivisión en varias fases, que coinciden con una estructura tipo de intervención en bienes culturales (ver Tabla I, última columna) (Instituto Andaluz del Patrimonio Histórico, sin fecha).

No obstante, los problemas se presentaron de manera desestructurada, con diferentes posibles enfoques de solución, para aumentar la motivación y las oportunidades de análisis del alumnado (Morales Bueno, 20I8, p. 97). De la misma forma, este planteamiento respondía a los propósitos didácticos de ilustrar principios, conceptos y procedimientos y fomentar el razonamiento y la comprensión de los mismos (Duffy y Cunningham, I996, p. 193). Así, se programaron problemas reales de nuestro ámbito profesional y directamente frente a la obra.

Los alumnos desarrollaron cuatro bloques de problemas para articular la restauración del retablo de la ermita de Valme. De esta manera, afrontaron la restauración desde el primer día, es decir, el contexto motivacional fue apremiante (Biggs, 2005, pp. 253-254). El tutor distribuyó los grupos por parejas cuya composición se realizó de forma aleatoria para evitar el sesgo de grupos ya preestablecidos por sus relaciones 
personales y para favorecer un contexto de aprendizaje colaborativo. El trabajo en equipo con aprendizaje cooperativo es, para Delgado y de Justo (2018, p. 197), uno de los factores más positivos y que apunta al principio de interdependencia positiva.

Al inicio de cada fase de intervención, se enunció el problema y se aclararon conceptos resolviendo determinadas dudas, interactuando con el tutor y los compañeros, como los indicativos visuales de algunas patologías (diferencias entre grietas por movimientos naturales de la madera o grietas por deficiencias constructivas), beneficios y desventajas de aditivos en los adhesivos, revisiones del concepto de reversibilidad de los tratamientos, etc. Los alumnos fueron articulando una base de conocimiento que evaluaron y verificaron (mayor o menor efectividad de los tratamientos). Se discutieron otras intervenciones de restauración de la misma tipología, localizando estos recursos. El ambiente cooperativo y su participación activa los hizo responsables del propio proceso de aprendizaje: investigando, aplicando y resolviendo.

Aunque el tutor eludió el planteamiento de problemas con solo una posibilidad correcta, tuvo que estar alerta para evitar la tentación de una dirección del conocimiento tradicional. A medida que la intervención y los conocimientos avanzaban, la figura del tutor se vio diluida. No obstante, en los primeros pasos fue necesaria una mayor presencia para impedir procesos deficientes, puesto que estaban trabajando sobre una pieza real. Así, la figura del tutor de prácticas se adapta de forma natural al cambio paradigmático de rol docente que el ABP dispone.

Tabla 2. Secuencia de problemas planteados y desarrollo de actividades.

\begin{tabular}{|c|c|}
\hline Problemas & Actividades desarrolladas \\
\hline \multirow{5}{*}{ Problema 1} & Documentación gráfica (mapas de daños) \\
\hline & Documentación fotográfica \\
\hline & Test de limpieza \\
\hline & Test de fijación y consolidación \\
\hline & Determinación de niveles de limpieza \\
\hline \multirow{6}{*}{ Problema 2} & Limpieza de depósitos superficiales \\
\hline & Fijación de los estratos de pintura. \\
\hline & Limpieza de reintegraciones anteriores alteradas \\
\hline & Limpieza del dorado \\
\hline & Sellado de grietas \\
\hline & Adhesión de piezas despegadas \\
\hline \multirow{3}{*}{ Problema 3} & Reintegración de la capa de preparación \\
\hline & Reintegración cromática \\
\hline & Protección final \\
\hline \multirow[b]{2}{*}{ Problema 4} & Programa de divulgación en redes sociales \\
\hline & $\begin{array}{l}\text { Programa de transmisión del conocimiento en revistas } \\
\text { especializadas }\end{array}$ \\
\hline
\end{tabular}


 \\ UN ENFOQUE DEL APRENDIZAJE BASADO EN PROBLEMAS (ABP) COMO METODOLOGÍA DIDÁCTICA EN LAS PRÁCTICAS EXTRACURRICULARES DEL GRADO... \\ ANTONIO J. SÁNCHEZ FERNÁNDEZ}

A continuación, se detallan los cuatro bloques de problemas propuestos para abordar la didáctica de la conservación-restauración con metodología ABP del retablo. Los cuatro problemas se corresponden con las fases de un proceso de intervención sobre un bien cultural. De esta manera, se sintetiza la metodología de intervención para la capacitación profesional del alumnado. Igualmente, cada bloque sigue un mismo patrón en el que se plantea: un tipo de problema (dilema o de aplicación), estudios autodirigidos por el alumnado, análisis de la información obtenida y evaluación de los resultados.

\section{I. Problema I. Se corresponde con los estudios previos y los criterios de intervención}

Primera fase: Se les planteó el problema tipo dilema: la conservación del retablo tal y como nos ha llegado a nuestros días o la eliminación de elementos posteriores para buscar un estado primitivo de autenticidad (elección de los criterios de intervención). El problema encarnaba cierta ambigüedad dirigida, con el fin de explorar matices en los criterios de intervención y huir de la sistematización automática de los trabajos de restauración. Se les pidió que organizaran conceptos de la disciplina. Así, trabajaron con las ideas de autenticidad; pátina noble frente a daños y alteraciones; métodos de documentación de bienes culturales, o tipos de disolventes.

Segunda fase: Los alumnos realizaron una serie de estudios autodirigidos. Investigaron la historia material del retablo, es decir, el conjunto de sucesos que definen la trayectoria material del bien; analizaron el estado de conservación del retablo de forma crítica, buscando las causas en origen de las patologías; registraron el cuadro patológico con gráfico de daños, y determinaron los límites de algunas operaciones como la limpieza técnica. Hay que destacar el uso de nuevas tecnologías aplicadas al análisis del estado de conservación. Una de las patologías que los alumnos registraron fueron las colonizaciones biológicas. En el reverso del retablo aparecieron restos óseos de animales que fueron analizados e identificados con Google Lens gracias a la estructura del cráneo.

Tercera fase: Análisis, discusión y aplicación de la información obtenida. Los alumnos descubrieron que el retablo está compuesto con varias piezas de distintos retablos para adaptarlo al espacio de la ermita; que se utilizaron molduras para cegar un hueco destinado a albergar un sagrario y que la hornacina central estaba suplementada para modificar la proporción de la misma; que esta adaptación y otra intervención anterior en los años 90 se asocian a un grupo de patologías concretas y localizadas; que la yuxtaposición de las piezas constituyentes y el proceso de adaptación al espacio de la ermita constituyen parte de la autenticidad del bien en cuestión; determinaron el conjunto de disolventes con los parámetros de toxicidad, penetración y volatilidad idóneos y el procedimiento de limpieza.

Cuarta fase: evaluación de los resultados. Cada alumno evaluó directamente con el tutor los resultados obtenidos en el propio retablo y cómo estos predisponían y facilitaba la introducción a la siguiente fase de intervención y/o cómo influían en la autenticidad material de la obra. 
UN ENFOQUE DEL APRENDIZAJE BASADO EN PROBLEMAS (ABP) COMO METODOLOGÍA DIDÁCTICA EN LAS PRÁCTICAS EXTRACURRICULARES DEL GRADO... ANTONIO J. SÁNCHEZ FERNÁNDEZ

5.2. Problema 2. Se corresponde con los procedimientos y técnicas de conservación

Primera fase: Se planteó un problema de aplicación. En la fase anterior habían realizado un análisis de los factores que influyen en el estado de conservación. En consecuencia, hubo que emplear un programa de tratamientos que garantizara la óptima conservación del retablo.

Figura i. Detalle del proceso de trabajo.

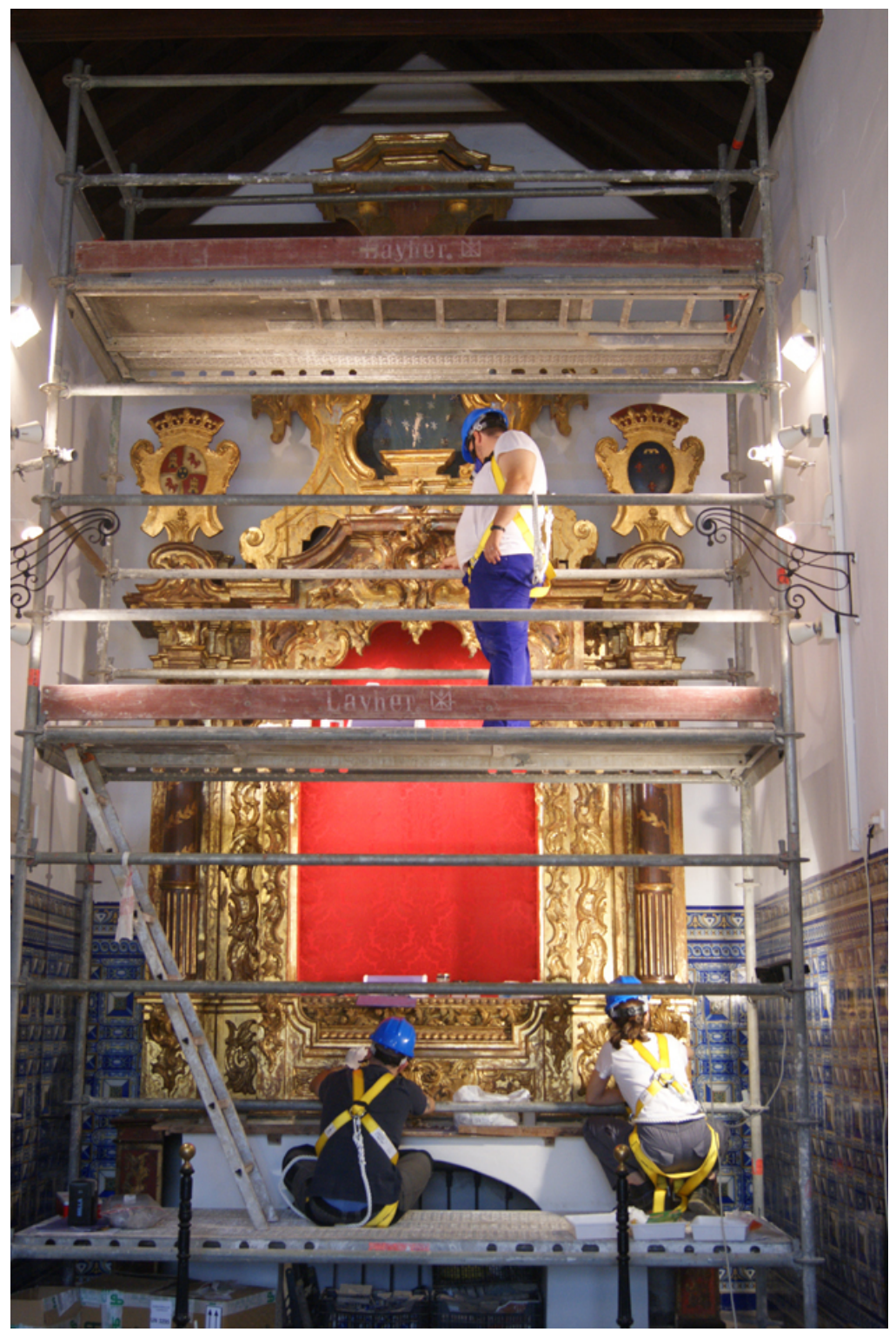




\section{DIDÁCTICA EN LAS PRÁCTICAS EXTRACURRICULARES DEL GRADO.. ANTONIO J. SÁNCHEZ FERNÁNDEZ}

Segunda fase: Estudio autodirigido. Con el conocimiento adquirido del problema I, tuvieron que intervenir la restauración directa del retablo de forma global. En los tratamientos más abiertos, con varias soluciones posibles, los alumnos aportaron los conocimientos que ya poseían y cada grupo elaboró un listado de diferentes enfoques, valorando la idoneidad de los mismos, según los objetivos de aprendizaje que planteaba el problema. En determinados momentos, los alumnos no supieron progresar con sus propios conocimientos. De esta forma, tuvieron que definir qué aspectos necesitaban para resolver la coyuntura. Estas cuestiones tenían que ver principalmente con la secuencia de estratos aplicados en la intervención de los años 90. La limpieza química no resultaba uniforme por lo que dedujeron cómo estaba articulada dicha intervención.

Tercera fase: Análisis, discusión y aplicación de la información obtenida. Con los estudios anteriores discutieron la posible naturaleza de los estratos observados (grasa o magra) y redefinieron el sistema de limpieza aplicando las nuevas premisas. Resultó fundamental proporcionar un tiempo para el análisis propiciando el interés para la investigación y su discusión, dando un lugar a cada uno para exponer su punto de vista.

Cuarta fase: evaluación de los resultados. Cada alumno evaluó directamente con el tutor los resultados obtenidos en el propio retablo y cómo estos influían en la autenticidad del bien. Comenzaron a establecer la sensibilidad suficiente para determinar, por sí mismos, hasta dónde podían limpiar sin provocar daños.

\subsection{Problema 3. Se corresponde con los procedimientos y técnicas de restauración}

Primera fase: Al igual que el problema anterior, se planteó un problema de aplicación. Todos los conocimientos adquiridos anteriormente tenían un carácter acumulativo y encadenado, y en esta fase volvían a tener que aplicar un programa de tratamientos que garantizara la óptima restauración del retablo.

Segunda fase: Estudio autodirigido. Otro de los aspectos fue la búsqueda de información externa por parte de los alumnos, en forma de casos prácticos de otros retablos, con la exposición de tratamientos realizados o criterios de intervención aplicados. Igualmente, en la restauración directa del retablo, los alumnos no supieron progresar con sus propios conocimientos en momentos concretos. Volvieron a definir qué aspectos necesitaban para resolver el problema. Los principales obstáculos los encontraron en la aplicación de las técnicas para la diferenciación entre la zona original y la reintegración cromática.

Tercera fase: Análisis, discusión y aplicación de la información obtenida. Tras la reflexión de los factores que influían en la percepción del retablo, incluyeron la variable de uso litúrgico vigente. En este sentido, optaron por reproducir el estrato de embolado con una tinta plana y la capa de dorado con rigatino (rayado).

Cuarta fase: Evaluación de los resultados. Cada alumno evaluó directamente con el tutor los resultados obtenidos en el propio retablo. Pudieron comprobar la eficacia de la reintegración cromática en la construcción de la unidad estética de la pieza.

\subsection{Problema 4. Se corresponde con la difusión y divulgación de una intervención}

Primera fase: Presentamos un problema de aplicación. Se planteó la actividad de difusión y divulgación de la restauración. 
UN ENFOQUE DEL APRENDIZAJE BASADO EN PROBLEMAS (ABP) COMO METODOLOGÍA DIDÁCTICA EN LAS PRÁCTICAS EXTRACURRICULARES DEL GRADO... ANTONIO J. SÁNCHEZ FERNÁNDEZ

Segunda fase: En esta fase se diseñó una estrategia de difusión y divulgación de las actividades desarrolladas, atendiendo a las fases de intervención y el permiso y complicidad de la propiedad del retablo.

Tercera fase: En esta etapa, se analizaron la importancia de la gestión de la información, el cuidado de la imagen como reflejo de los trabajos de restauración y la fluidez de comunicación con el cliente. Asimismo, se reflexionó sobre el valor de la presencia digital como profesionales liberales.

Cuarta fase: La evaluación de los resultados se produjo con el reflejo de visitas, seguidores, reenviado de información o comentarios de los usuarios de Internet.

Figura 2. Ejemplo de divulgación en redes sociales.

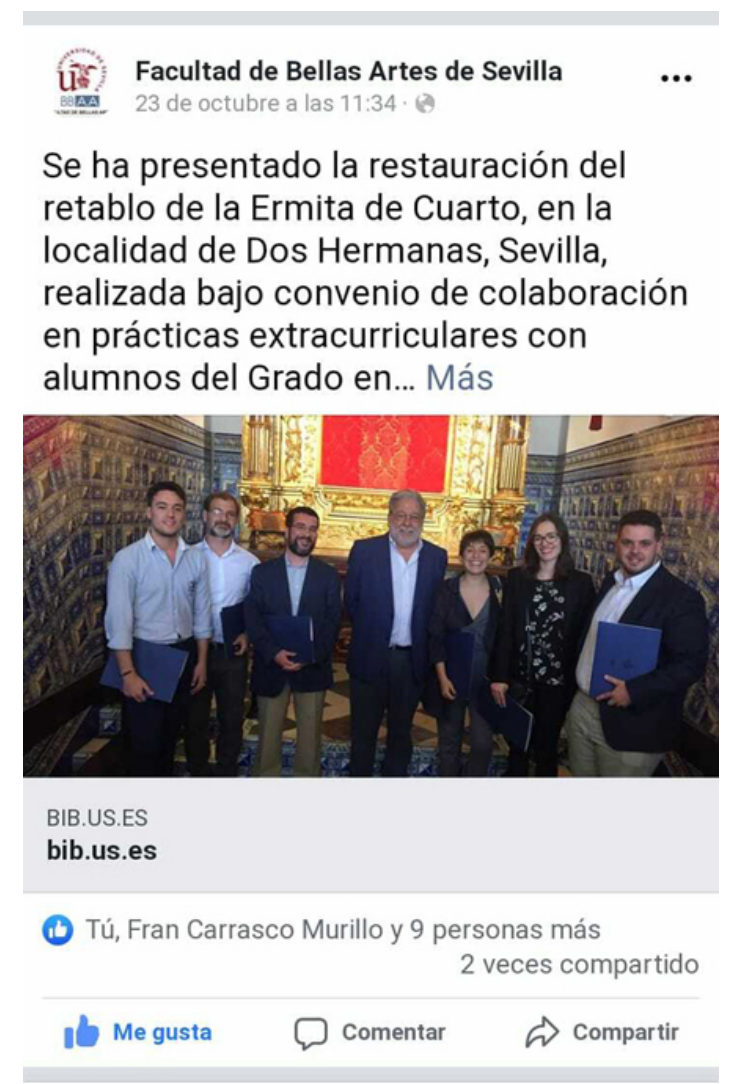

\section{Resultados y conclusiones}

Una manera óptima de convertirse en restaurador profesional es siendo y actuando como tal. Así, en el camino de resolución de los problemas planteados, los alumnos adquirieron destrezas autodirigidas que les acompañarán como restauradores profe- 


\section{DIDÁCTICA EN LAS PRÁCTICAS EXTRACURRICULARES DEL GRADO... ANTONIO J. SÁNCHEZ FERNÁNDEZ}

sionales. Por lo tanto, esta práctica evidenció la importancia de contar con modelos educativos que entrenen las competencias del profesional de la conservación-restauración. No solo en el desarrollo de técnicas de intervención, también en habilidades de elaboración de juicios críticos a partir del conjunto de condicionantes que presenta un bien cultural (como la aplicación de los criterios de intervención) o la toma de decisiones a partir del análisis y la ponderación de valores culturales de dichos bienes (elección y alcance de tratamientos).

Los problemas propuestos fueron cuestiones que forzaron a los alumnos a descubrir inductivamente la influencia de la historia material (el conjunto de sucesos que constituyen la trayectoria material de un bien cultural) y de factores extrínsecos e intrínsecos en el estado de conservación del retablo. De igual forma, dicho planteamiento trató de ejercitar habilidades cognitivas de razonamiento, análisis y síntesis para la elección del procedimiento más adecuado de intervención sobre el retablo, además de la toma de decisiones del criterio de intervención para no desvirtuar sus valores culturales. Asimismo, durante la práctica, el aprendizaje adquirido se pudo replicar en otras zonas con las mismas patologías (distinción de indicativos visuales de alteración), por lo que se desarrollaron destrezas de autosupervisión.

Durante el proceso de intervención sobre el retablo con una metodología ABP, además del aprendizaje en materia de conservación-restauración, los alumnos proyectaron un análisis de sus propias necesidades formativas. Puesto que las prácticas tenían como objetivo la aproximación al mundo laboral, dichas necesidades tendían a satisfacer su faceta como futuros profesionales. Así, diagnosticaron una carencia en la creación, gestión y difusión del currículo personal y estrategias de búsqueda de empleo por cuenta propia. No obstante, también detectaron un déficit en el uso de nuevas tecnologías óptimas para la gestión de la documentación del patrimonio cultural y para la investigación y transferencia de resultados académicos.

Este pensamiento crítico, interiorizado por los alumnos, nos coloca en un flujo cíclico de aprendizaje, en el que, detectados estos «nuevos problemas», se hace necesaria la programación de otros proyectos formativos para solventar las necesidades manifestadas por los propios alumnos. No obstante, esto evidencia el valor del ABP no solo como metodología para el desarrollo de capacidades curriculares, sino también para fomentar habilidades relacionadas con la empleabilidad y la convivencia (Luy-Montejo, 20I9).

Otro posible obstáculo consiste en ponderar la estructuración planificada de una intervención de restauración en bienes culturales con la inclinación desestructurada a la que tiende el método ABP. Así, la figura del tutor, que debe calibrar y monitorizar estas acciones, se reivindica en esta posición, evitando riesgos que la propia práctica pudiera ejercer sobre la conservación del bien. Del mismo modo, entendemos que el tutor debe tener una especial sensibilidad hacia cuestiones socioeducativas, además del conocimiento básico de la metodología científica y de investigación en materia de restauración.

Asimismo, coincidimos con la demanda de Prado y Zambrana (2017, p. 6I), reivindicando medidas determinadas (seguro de responsabilidad civil y un seguro de accidentes específico), atendiendo a las peculiaridades de los trabajos de restauración.

Por otro lado, las encuestas estandarizadas proporcionadas por ICARO (plataforma interuniversitaria que gestiona la información, oferta e inscripción de las prácticas) y 
UN ENFOQUE DEL APRENDIZAJE BASADO EN PROBLEMAS (ABP) COMO METODOLOGÍA DIDÁCTICA EN LAS PRÁCTICAS EXTRACURRICULARES DEL GRADO.. ANTONIO J. SÁNCHEZ FERNÁNDEZ

que se formalizaron al final de la práctica describieron una serie de cuestiones a modo de evaluación general de la actividad de enseñanza-aprendizaje:

- Autopercepción de adquisición de competencias profesionales en materia de restauración.

- Mejora en el interés y motivación en la intervención de restauración.

- Desarrollo de habilidades para el trabajo en equipo, de forma colaborativa.

El enfoque ABP se presenta como una adecuada metodología de enseñanza-aprendizaje de la conservación-restauración, ya que consigue abordar las cuestiones profesionales futuras de los alumnos. En la experiencia descrita, el trabajo en equipo para la restauración del retablo ha permitido que cada uno de los individuos haya construido un marco de aprendizaje significativo, con procesos que han desarrollado competencias específicas de la profesión y con un resultado final óptimo. Hay que destacar que la restauración se realiza en un contexto real, lo que implica salir del aula y definir una investigación de campo.

Figura 3. Resultado final.

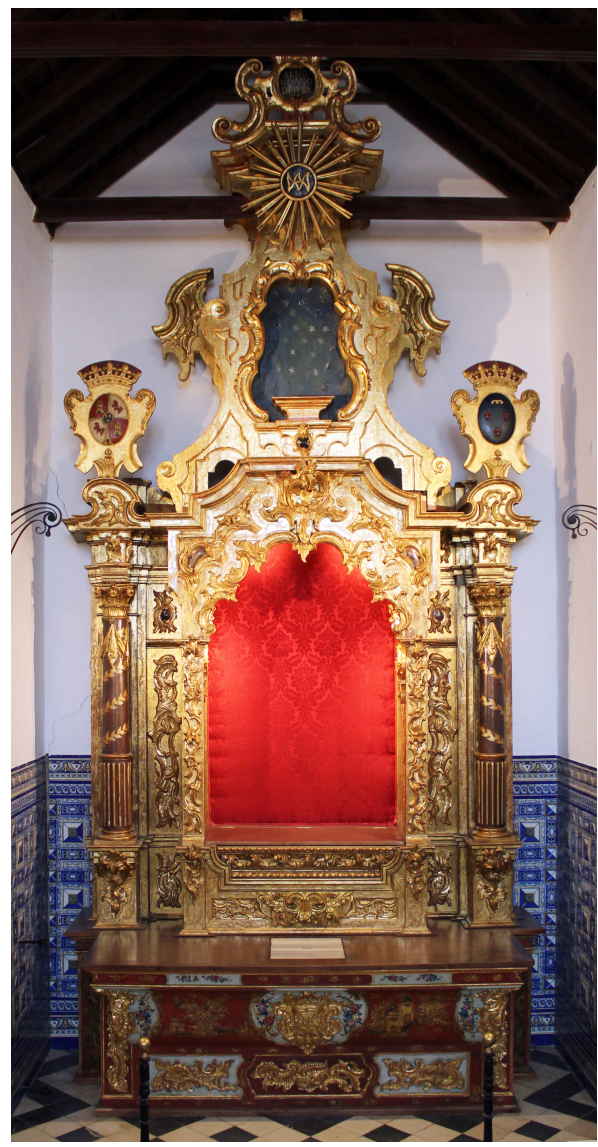


UN ENFOQUE DEL APRENDIZAJE BASADO EN PROBLEMAS (ABP) COMO METODOLOGÍA DIDÁCTICA EN LAS PRÁCTICAS EXTRACURRICULARES DEL GRADO... ANTONIO J. SÁNCHEZ FERNÁNDEZ

\section{Bibliografía}

Barrows, H. S. (1986). A taxonomy of problem-based learning methods. Medical Education, 20, 48I-486.

Biggs, J. (2005). Calidad del Aprendizaje Universitario. Madrid: Narcea Ediciones.

Boud, D. (1985). Problem-based Learning in Education fot the Professions. Sydney: Higher Education Research and Development Society of Australasia.

Brandi, C. (2007). Teoría de la Restauración. Madrid: Alianza Editorial.

Delgado, A. y De Justo, E. (20I8). Evaluación del diseño, proceso y resultados de una asignatura técnica con aprendizaje basado en problemas. Educación XXI, 2I(2), I79-203. DOI: https://doi.org/I0.5944/educxxi.19415

Duffy, T. M. y Cunningham, D. J. (1996). Constructivism: Implications for the design and delivery of instruction. En D. H. Jonassen (de.), Handbook of research for educational communications and technology (pp. 170-198). New York: Macmillan.

Gewurtz, R. E.; Coman, L.; Dhillon, S.; Jung, B. y Solomon, P. (2016). Problem-based learning and Theories of Teaching and learning in Health Professional Education. Journal of Perspectives in Applied Academic Practice, 4(I), 59-70. DoI: https://doi.org/Io.I4297/ jpaap.v4ir.194

Instituto Andaluz del Patrimonio Histórico. (sin fecha). Conocer para intervenir: criterios, métodos y técnicas. Recuperado el 4 de octubre de 20I9, de: https://www.juntadeandalucia.es/organismos/culturaypatrimoniohistorico/iaph/areas/conservacion-restauracion/ conocer-intervenir.html.

Luy-Montejo, C. (2019). El Aprendizaje Basado en Problemas (ABP) en el desarrollo de la inteligencia emocional de estudiantes universitarios. Propósitos y Representaciones, 7(2). DOI: http://dx.doi.org/ı0.205II/pyr20I9.v7n2.288

Marra, R.; Jonassen, D. H.; Palmer, B. y Luft, S. (20I4). Why problem-based learning works: Theoretical foundations. Journal on Excellence in College Teaching, 25(3-4), 22I-238. Recuperado el 25 de enero de $202 \mathrm{I}$, de http://celt.miamioh.edu/ject/issue.php?v=25\&n=3\%20 and $\% 204$.

Molina, E.; Bolívar, A.; Burgos, A.; Domingo, J.; Fernández, M.; Gallego, M. J.; Iranzo, P.; León, M. J.; López, M. C.; Molina, M. A.; Pérez, P. y Ponce, C. (2004). La mejora del Practicum, esfuerzo de colaboración. Profesorado: Revista de Currículum y Formación del Profesorado, 8(2), I-3I. Recuperado el in de noviembre de 2019, de http://www.ugr. es/ recfpro/rev82ART 4 .pdf.

Morales Bueno, P. (20I8). Aprendizaje Basado en problemas (ABP) y habilidades de pensamiento crítico ¿una relación vinculante? Revista Electrónica Interuniversitaria de Formación del Profesorado, 2I(2), 9I-I08. DOI: http://dx.doi.org/Io.6or8/reifop.2I.2.32337I

PÉrez, L. (20I8). El aprendizaje basado en problemas como estrategia didáctica en educación superior. Voces de la Educación, 3(6), I55-167. Recuperado el 25 de enero de 2021, de https:// www.revista.vocesdelaeducacion.com.mx/index.php/voces/article/view/r27.

Prado-Campos, B. y Zambrana-Vega, M. D. (2017). Teaching innovation: the management and development of extracurricular activities in the field of cultural object conservation and restoration of wall painting. Chapel of San Andrés (Seville-Spain). REIRE Revista d'Innovació $i$ Recerca en Educació, Io(2), 52-63. DoI: http://doi.org/Io.1344/reire2017.10.217435

Restrepo Gómez, B. (2005). Aprendizaje basado en problemas (ABP): una innovación didáctica para la enseñanza universitaria. Educación y educadores, 8, 9-20.

Rodríguez-Hidalgo, A. J.; Calmaestra Villén, J. y Maestre Espejo, M. (20I5). Desarrollo de competencias en el práctico de maestros: ABP y coaching multidimensional. Profesorado: Revista de Currículum y Formación del Profesorado, Ig(I), 4I4-434. Recuperado io de octubre de 20I9, de http://www.ugr.es/local/recfpro/revigiCOL8.pdf. 
UN ENFOQUE DEL APRENDIZAJE BASADO EN PROBLEMAS (ABP) COMO METODOLOGÍA

DIDÁCTICA EN LAS PRÁCTICAS EXTRACURRICULARES DEL GRADO...

ANTONIO J. SÁNCHEZ FERNÁNDEZ

SANTOS GiL, H. (2009, octubre 17). Ciento cincuenta aniversario de la restauración de la ermita de Valme. $A B C$ de Sevilla. Recuperado el 25 de enero de 202I, de https://sevilla.abc.es/cultura/ sevi-ciento-cincuenta-aniversario-restauracion-ermita-valme-200910170300-113720797954_ noticia.html.

Universidad de Sevilla. (2019). Portal de estudio y acceso al Grado de Conservación y Restauración de Bienes Culturales de la Universidad de Sevilla. Datos generales, Objetivos y Competencias. Recuperado el 4 de octubre de 20I9, de http://www.us.es/estudios/grados/ plan_193? $\mathrm{p}=4$. 
